\title{
A workcell calibration method for enhancing accuracy in robot machining of aerospace parts
}

\author{
Francesco Leali • Alberto Vergnano • Fabio Pini • \\ Marcello Pellicciari • Giovanni Berselli
}

Received: 15 November 2013 / Accepted: 10 April 2014 / Published online: 12 June 2014

(C) Springer-Verlag London 2014

\begin{abstract}
Industrial robotics provides high flexibility and reconfigurability supported by a user-friendly programming, but still lacks in accuracy. An effective workcell calibration reduces errors in robot manufacturing and enables robot machining applications. A novel workcell calibration method is embedded in an integrated design framework for an in-depth exploitation of CAD-based simulations and offline programming. The method is composed of two steps: first calibration of the workpiece-independent equipment in the workcell layout and final automated online calibration of workpiecedependent equipment. The method is finally applied to a changeable robotic workcell for finishing aluminium cast housings for aerospace gear transmissions characterised by complex shapes and by close dimensional and geometrical specifications. Experimental results prove the method effectiveness in enhancing accuracy in robot machining.
\end{abstract}

Keywords Workcell calibration · Industrial robotics · Integrated design $\cdot$ Aerospace industry

Electronic supplementary material The online version of this article (doi:10.1007/s00170-014-6025-y) contains supplementary material, which is available to authorized users.

F. Leali $\cdot$ A. Vergnano $\cdot$ F. Pini $\cdot$ M. Pellicciari $(\bowtie) \cdot G$. Berselli

"Enzo Ferrari" Engineering Department, University of Modena and

Reggio Emilia, via Vignolese 905/B, 41125 Modena, Italy

e-mail: marcello.pellicciari@unimore.it

F. Leali

e-mail: francesco.leali@unimore.it

A. Vergnano

e-mail: alberto.vergnano@unimore.it

F. Pini

e-mail: fabio.pini@unimore.it

G. Berselli

e-mail: giovanni.berselli@unimore.it

\section{Introduction}

Mechanical parts in aerospace industry are characterised by complex shapes and narrow tolerance ranges to accomplish light weight design requirements and comply with standards and safety regulations. Finishing their complex profiles generally requires 5 axes computer numerical control (CNC) tool machines with huge investment costs and too long setup times for lot changeover.

Compared to $\mathrm{CNC}$ tool machines, industrial robots offer a greater dexterity within extended work envelopes [1] so that changeable robotic workcells represent an effective solution from an industrial point of view, as demonstrated by [2-4]. As clarifying example, Table 1 reports cycle times and production rates for the automated machining of an aluminium automotive brake caliper. The machining cycle consists of several operations (i.e., milling, contouring, drilling, and chamfering) realised on all the part sides through a multi-(re)positioning on customised fixtures. Following a traditional approach based on CNC machining, feeding, part (re)positioning and inspection are manually performed by specialised operators. Conversely, a vision-guided automated feeding and an online inspection complete the robotic manufacturing cycle. Cycle time is the sum of the time needed to fixture the brake caliper (setup time), the contact time between the tools and the workpiece during machining (cutting time) and the time spent for part inspection (part inspection time). Productivity, expressed in parts per day, returns the hourly productivity for a given production rate. Thanks to its full automation, two working shifts are considered for the changeable robotic workcell. The labour cost and equipment cost, in euros per part, are finally considered to give a rough evaluation of the investment efforts in terms of human work and equipment.

In industrial robotics, the workcell changeability, defined as the ability to cope with change or uncertainty, represents a key factor for small lot production of complex-shaped parts 
Table 1 Cycle times and production rates for machining an aluminium automotive brake caliper by a standard CNC tool machine and a changeable robotic workcell

\begin{tabular}{|c|c|c|c|}
\hline \multicolumn{2}{|l|}{ Production data } & \multirow{2}{*}{$\begin{array}{l}\text { CNC tool machine } \\
50.0\end{array}$} & \multirow{2}{*}{$\begin{array}{l}\text { Changeable robotic workcell } \\
32.8\end{array}$} \\
\hline Cycle time & $(\min )$ & & \\
\hline Setup time & $(\min )$ & $14^{\mathrm{a}}$ & $3^{\mathrm{b}}$ \\
\hline Cutting time & $(\min )$ & 18 & 25 \\
\hline Part inspection time & $(\min )$ & 18.0 & 4.8 \\
\hline Productivity & (parts/day) & 8 & 32 \\
\hline Hourly productivity & (parts/h) & 1 & 2 \\
\hline Production rate & (h/day) & 8 & 16 \\
\hline Labour cost per part & (€/part) & 11.34 & 0.06 \\
\hline Labour cost & $(€ / \mathrm{h})$ & 20 & 20 \\
\hline Labour time & (min/part) & $34^{\mathrm{c}}$ & $0.18^{\mathrm{d}}$ \\
\hline Equipment cost per part & $(€ /$ part $)$ & 18.8 & 3.6 \\
\hline
\end{tabular}

${ }^{\text {a }} 7 \min \times 2$ (re)positioning for each part

b 3 min for loading a pallet with 15 parts

${ }^{\mathrm{c}}$ Sum of setup time and control time

${ }^{\mathrm{d}}$ Setup time only

[5]. Changeability is enabled by the robot inherent flexibility, the workcell modularity and (re)configurability and by the overall system (re)programmability [6].

Changeability could be effectively addressed through specialised design frameworks based on a Product analysis, Process identification and Resource selection design (PPR), as described in $[7,8]$. Such approach, also named "robofacturing", is centred on the use of CAD-based environments and offline programming (OLP) tools to enable changes in the robot workcell layout in the settings of the mechatronic equipment and in the robot's tasks.

The first steps of robofacturing investigate the industrial problem and analyse the workpiece geometry and material, collecting all the data into a CAD model (product). Then, the machining cycle (process) is conceptually defined and detailed, addressing part poses, tools and machining parameters.

All the equipments needed to realise the machining process (resources) are designed and modeled within a customised CAD-based environment. Reference frames, envelopes, kinematics and control logics are so defined and associated to each resource. Robot target points are then generated through OLP and grouped in machining paths. Even if manual programming is widely diffused yet, OLP software, integrated by specific machining functions or dedicated packages, have demonstrated the capability to reduce the productivity loss at changeover [9]. Every machining path results as an independent module composed by a list of tasks, resources involved and robot code, giving to the workcell a fully changeable architecture. A workcell virtual prototype is then available to simulate and virtually optimise the machining performances and finally generate the workcell program. At the end, workcell calibration is performed. The workcell calibration is the action of matching the nominal and the actual poses of every device which enters into the definition of the robotic manufacturing cycle. A complete workcell calibration considers both the robot (absolute calibration) and the frames of the workpieces, tools and mechatronic devices (relative calibration) $[10,11]$.

The next section introduces a brief review of the main error sources decreasing the robot accuracy and a review of the approaches for workcell calibration proposed in the scientific literature. Section 3 describes an automated workcell calibration method developed to reduce errors in robotic manufacturing and extend the use of industrial robots to machine complex aerospace parts. Section 4 presents and discusses the results of a verification case study.

\section{Error compensation in robot machining}

Among the many sources of errors of machine tools, thermal deformation and geometric errors have been traditionally known as key contributors, also contributing for $70 \%$ of workpiece errors in precision machining. Nowadays, after the geometric and thermal errors are compensated for, cutting force-induced errors become the major source of machine tool errors. For example, a $500 \mathrm{~N}$ cutting force during milling operation will cause $0.02-\mathrm{mm}$ error for a CNC machine [12]. The accuracy provided by the structure of a standard CNC, which has a stiffness over $30 \mathrm{~N} / \mu \mathrm{m}$, cannot be replicated by the open kinematic chain of industrial robots since a largesized articulated robot IRB6400, for instance, has a stiffness around $0.5 \mathrm{~N} / \mu \mathrm{m}$. Moreover, several inaccuracy factors affect robotic workcells, mainly due to workpiece geometric and 
dimensional tolerances (Product), to machining (process) and due to the inherent inaccuracy of the robot itself and of the mechatronic devices which composed the workcell (resources).

\subsection{Accuracy in robot machining}

\subsubsection{Product}

The dimensions and geometry of every part vary within its fields of tolerance. As a result, the relative position between the robot and the part is not repeatable in the production lot. For both part-on-robot configuration and tool-on-robot configuration, grasping fixtures are typically designed with wearable contact parts and customised reference pins (Fig. 1). The 3D sum of ISO 8062-CT7 tolerances on an aluminium cast part with an envelope of $290 \times 155 \times 80 \mathrm{~mm}$, grasped by a standard pneumatic gripper equipped by machined steel jaws and moved by the robot, ranges from 1 to $1.5 \mathrm{~mm}$ for every part. A reduction about $30 \%$ is reachable adopting a fixed fixture system since the part positioning is not influenced by the inherent robot inaccuracy [12]. In OLP, the relative position between the robot and the part is described by the Cartesian reference frames.

\subsubsection{Process}

Machining forces are time varying, and their magnitude is the function of the process parameters, such as spindle speed, axial depth of cut, radial depth of cut, material removal rate, lubrication conditions, chip load and part material [13]. In robotic machining process, due to the low stiffness of the industrial robot, the force-induced deformation of the robot structure is the single most dominant source of workpiece surface error. As an example, a $500 \mathrm{~N}$ cutting force during milling operation will cause a $1-\mathrm{mm}$ position error for a largesized articulated robot, e.g. a IRB6400 [12]. Figure 2 shows some large industrial robots adopted for machining.

Moreover, at specific parameter values, the robot TCP and the tool vibrate producing chatter. Chatter is one of the major reasons which prevent the wide use of robots for machining [14]. Finally, temperature variation of mechanical parts during machining [15] and working conditions (e.g. presence of swarf, chips, metal dust, etc.) also affect the final quality of machining.

When an OLP tool is adopted, the full correspondence between the real and the virtual controller implemented in the software guarantees an exact analysis of the robot's behaviour and suggests a possible partial correction of the robot trajectories to compensate the most important dynamic errors [2]. Anyway, errors directly connected to the machining process vary between 1 and $1.5 \mathrm{~mm}$.

\subsubsection{Resources}

The poor accuracy declared by the manufacturer for standard industrial robots is mainly caused by the low stiffness of the joints and geometric errors in robot manufacturing and assembly, clearances in motors and geared transmission mechanisms, such as for reducers, and backlash and bearing runout errors [16]. Backlash, in particular, yields to high torque impulses which can excite torsional vibrations. Moreover, link and joint compliance contribute up to $8-10 \%$ of the position and orientation errors of the tool centre point (TCP) [17]. According to the definition of repeatability and accuracy given by ISO 9283:1998, typical accuracy claimed for an industrial manipulator is about $\pm 1 \mathrm{~mm}[18,19]$, but values of $0.3 \mathrm{~mm}$ could be reached with accurate compensation [20]. Repeatability instead ranges in $0.1-0.03 \mathrm{~mm}$. Auxiliary mechatronic equipment is arranged in a reconfigurable machining workcell (Fig. 3).

Every auxiliary device introduces specific sources of errors due to its manufacturing and assembly tolerances and due to its positioning within the workcell layout, often roughly realised on the factory floor. During the workcell calibration, the exact position and orientation of every device that contributes to the manufacturing process have to be measured and corrected in the robot controller before starting the machining cycle. Such operation is normally done by a specialised operator. According to the ability of the operator, the error sum varies from 5 to $10 \mathrm{~mm}$ [12]. In OLP, reference frames are used to describe the nominal position of each device. Such frames are used by the robot controller to orient the robot TCP during the machining cycle.
Fig. 1 Product clamping for a part-on-robot configuration (left) and tool-on-robot configuration (right) (courtesy SIR SpA-Italy)
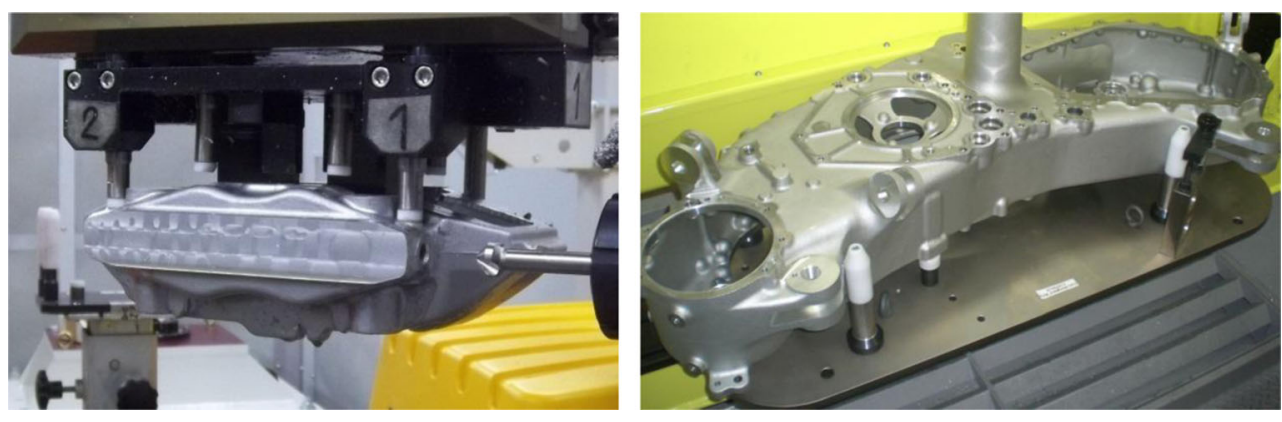

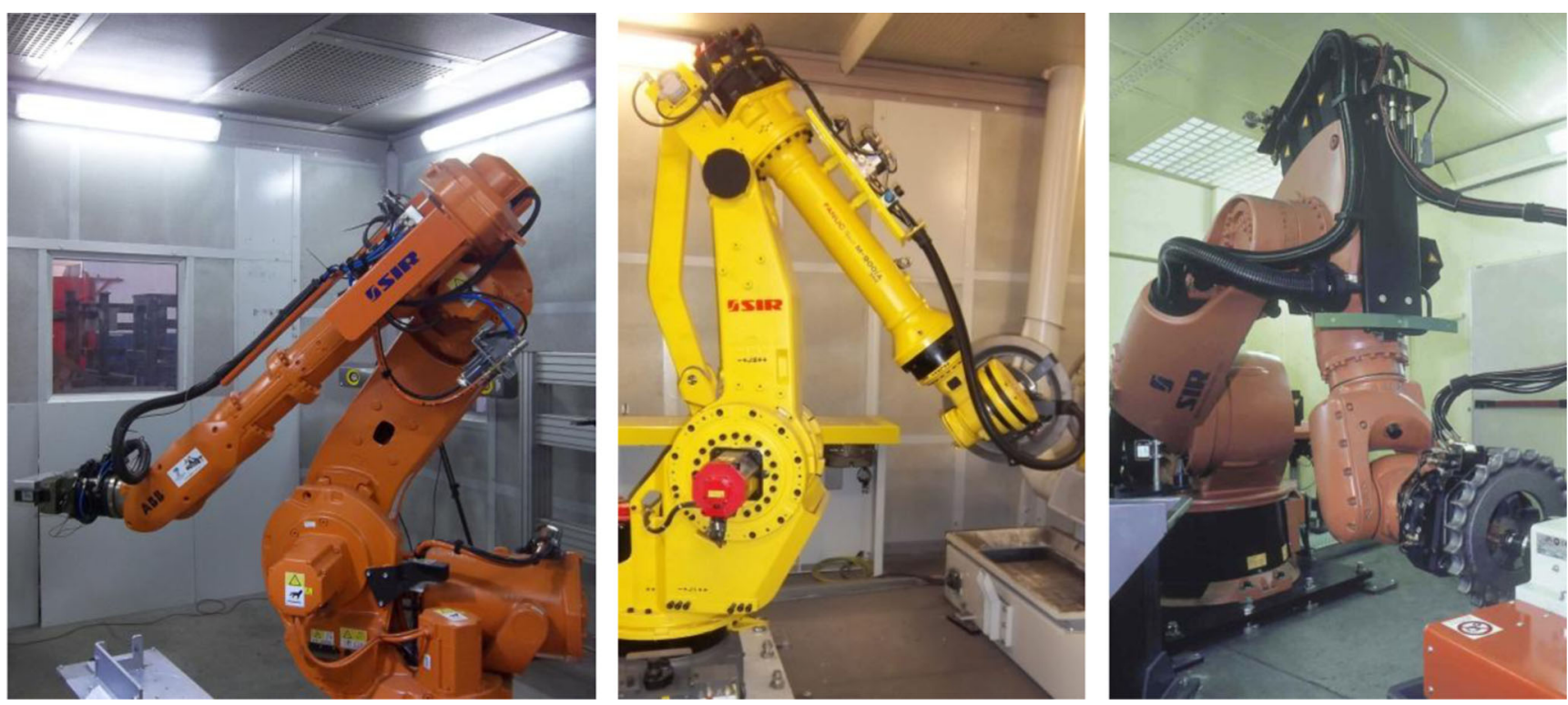

Fig. 2 Examples of industrial robots used for machining: ABB IRB 6640-185/2.8, FANUC M-900iA/350 and KUKA KR360-2 (courtesy SIR SpAItaly)

\subsection{Workcell calibration}

As reported in the previous section, the most evident and easily compensable source of errors is given by the measurement and correction of errors through the workcell calibration, especially when an OLP approach is followed. In fact, as already underlined, the OLP approach, when applied to the design or reconfiguration of changeable robotic workcells, introduces a group of reference frames which are used by skilled programmers to modularize the program architecture with the nominal position of each element of the robotic workcell. According to the common terminology used in industrial robotics [10] and as shown in Fig. 4, the world frame is the robot absolute reference. The base frame is the reference system which defines the robot zero position, and it is located on the fixed base of the robot. In a workcell with one robot only, the base frame matches with the world frame. The wrist frame is located on the robot wrist flange and defines the robot kinematic chain. Objects attached to the robot flange are referenced with specific frames. In case of spindle moved by the robot, a tool frame defines the position of every tool tip. The fixture frame is used to identify and locate stationary or movable fixtures with respect to the base frame. The workpiece frame defines the relation between the workpiece zero point and its relative fixture, while a target frame is used to describe the robot's configuration at every point of a work path. The robot movements are defined with a sorted sequence of matches between the tool frames and the target frames.

Errors in the definition of the reference frames in the robot controller have direct impact on the final accuracy. The robot's kinematic and dynamic errors depend on the difference between the actual dimensions of every robot link and their parameters stored in the controller, also referred as absolute positioning inaccuracy. The misalignment from nominal to actual poses of each element in the workcell layout contributes to the relative positioning error $[10,11]$. The most common absolute methods are the model-based parametric and nonparametric calibrations [21]. Model-based calibration improves the robot accuracy through a parametric identification of the main physical error sources. Model-based calibration is defined by $[22,23]$ three main levels: joint, kinematic and nonkinematic. The joint level calibration corrects the relationship between the signal produced by the transducer at every joint and its actual displacement, including drives and joints' sensors. The kinematic level calibration acts on the complete robot kinematic model, aiming at determining the basic
Fig. 3 Machining changeable robotic workcell (courtesy SIR SpA-Italy)

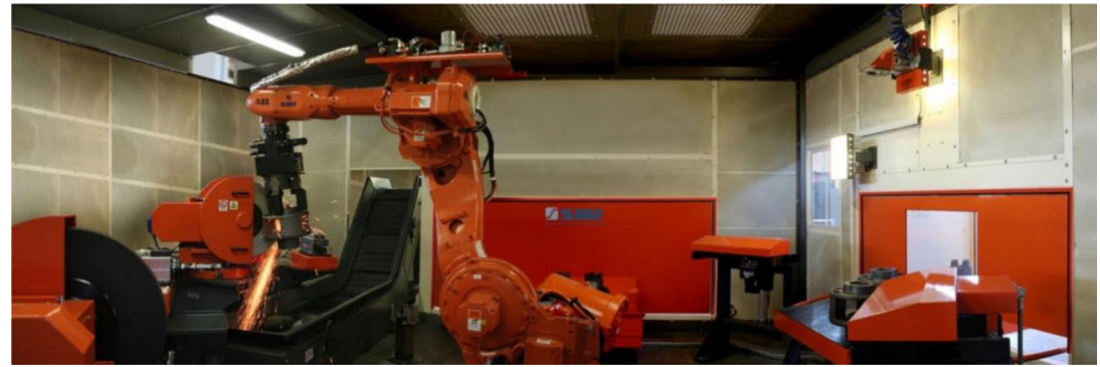




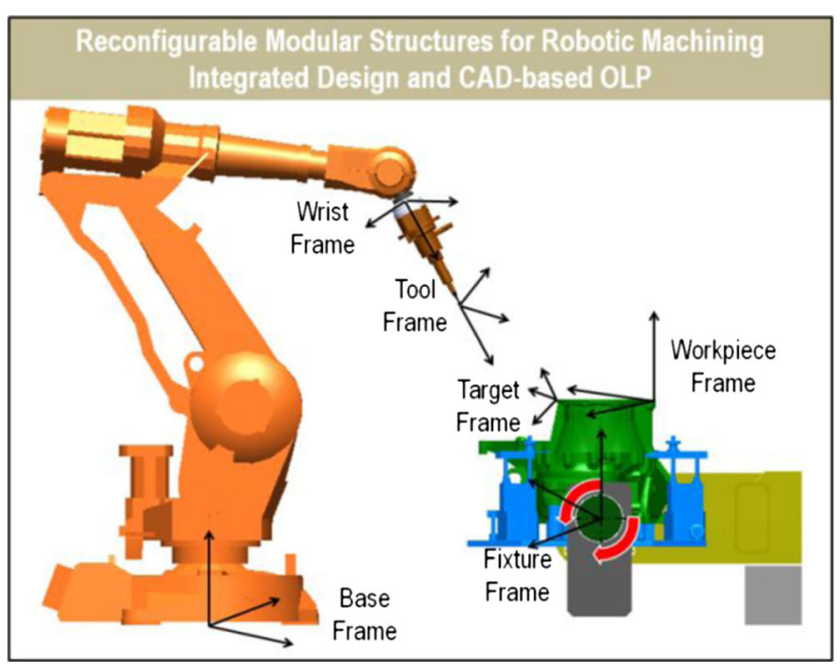

Fig. 4 Main reference frames in a changeable workcell for a spindle on robot configuration

kinematic geometry of the robot as well as the correct angles of every joint. The nonkinematic covers errors in robot positioning due to the dynamic effects, such as joint compliance, friction and clearances.

Nonparametric calibration estimates the robot's positioning errors through analytical interpolation methods which start from the measurement of the mechanical properties of the robot in predefined configurations. Absolute instruments can be effectively used for robot absolute calibration as laser trackers or 3D cameras [2, 20, 21]. Other error sources are related to dimensional and geometric variations of the elements, misalignments due to the workpiece feeding and pose, tool wearing and installation of devices [11].

A further classification of relative calibration methods is introduced by Gan et al. [24], where the calibration can adopt external instruments or use of the robot itself. Examples of external devices are coordinate measuring systems, which can be used to measure the position of the devices within the robotic workcell. This approach is particularly time- consuming and has to be applied every time the workcell configuration changes, which is frequent in case of small production batches. On the contrary, the robot itself can be used as a carrier for accurate measuring sensors. State-of-theart instruments and procedures are described in [25-27].

Relative calibration depends on the specific robotic workcell configuration and manufacturing application [10]. Even if many OLP developers and vendors (e.g. ABB, FANUC and DELCAM) offer solution based on simplified procedures within OLP tools, a full-integrated method for general applications, involving CAD-based and OLP environments, is not available.

\section{Workcell calibration method}

A specific method is here proposed to perform the workcell calibration through a flow of steps, including the reference frame definition during the behavioural virtual simulation. The method is part of a wider design framework. Before starting to model the tasks, the devices in the workcell are classified as workpiece-independent and workpiecedependent. The firsts must be configured, dimensioned or designed for the general robot operations and define the main architecture of the changeable workcell. The latters depend on the features of every single part code and are also influenced by their deviations from the nominal values. Positioners, robot(s) and tool racks are examples of workpieceindependent elements while fixtures, jigs, machining tools are dependent ones [28]. The calibration tasks related to these categories are then called by the controller main program with different purposes and timing.

The proposed workcell calibration method uses the robot itself to measure the elements of interest with an extra sensor mounted on the robot tool flange identified by the Wrist Frame. An additional stationary sensor measures the reference
Fig. 5 Workpiece-dependent and workpiece-independent elements in the calibration method

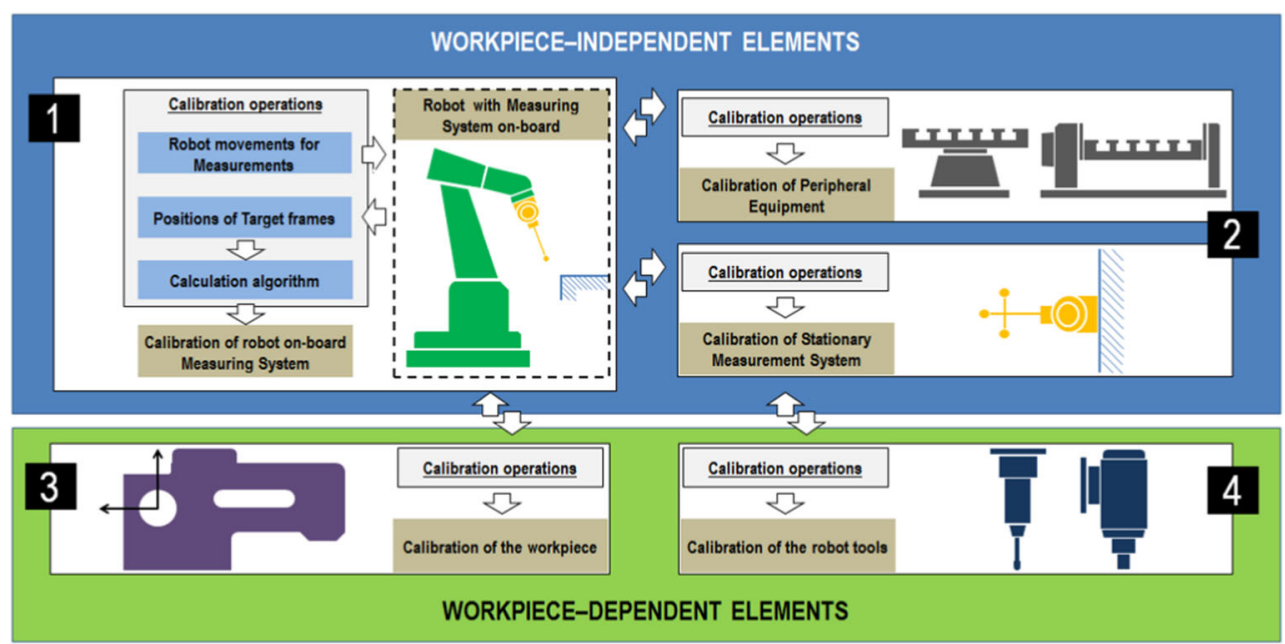


Fig. 6 Workpiece (a) and a detail before (b) and after (c) finishing

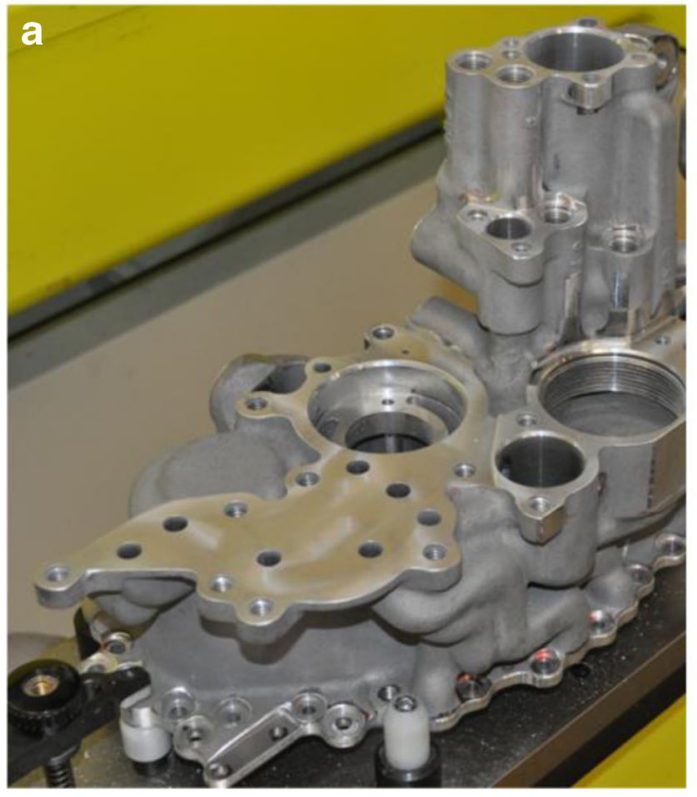

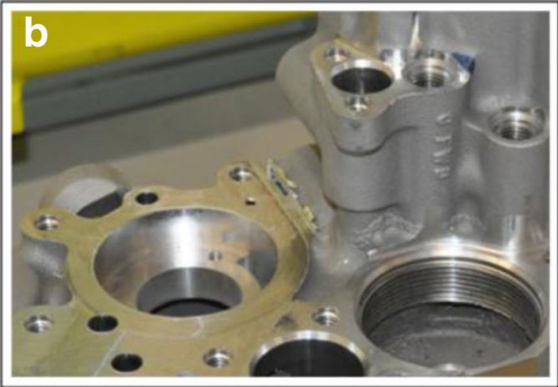

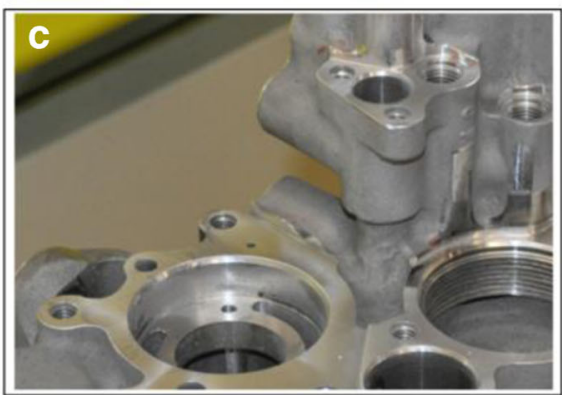

frames of the robot end effectors. The definition of the whole calibration process model follows four main steps, as outlined in Fig. 5.

Step 1 is the calibration of the robot measuring system to define the reference frame of the robot onboard sensor. The onboard sensor is moved against a fixed gauge block in the workcell. Whenever the sensor detects the block, a target frame is acquired by the robot controller, and the sensor tool frame is adjusted.

Step 2 consists in the calibration of the peripheral elements, including the workcell equipment and the stationary measuring system. The workcell equipment is directly measured by the robot onboard sensor. The stationary measuring system is then measured moving against it the same gauge block previously used, now handled by the robot. Steps 1 and 2 perform the workcell layout calibration once in a while. This means that they are run after the first workcell installation or reconfiguration and then, usually, every few days in case of low demanding application. Otherwise, shorter intervals are programmed, like few hours, for high parameters variations, as e.g. temperature gradients.

Step 3 is the workpiece alignment. More than the part location, the goal is to best fit the task paths that will follow on the pre-worked features, which are searched and detected by the robot and its onboard sensor.

Step 4 is the calibration of the robot tool using the stationary sensor to adjust the tool parameters and define the tool frames. The last two steps accomplish the workcell calibration and alignment. Step 3 must be run for each single processed workpiece while the frequency needed for step 4 is a tradeoff between productivity and machining quality. The method is implemented through the definition of parametric modules, including geometric characteristics and control logics for a quick set up in a CAD-based OLP.
Fig. 7 Reconfigurable robotic workcell layout in ABB RobotStudio environment

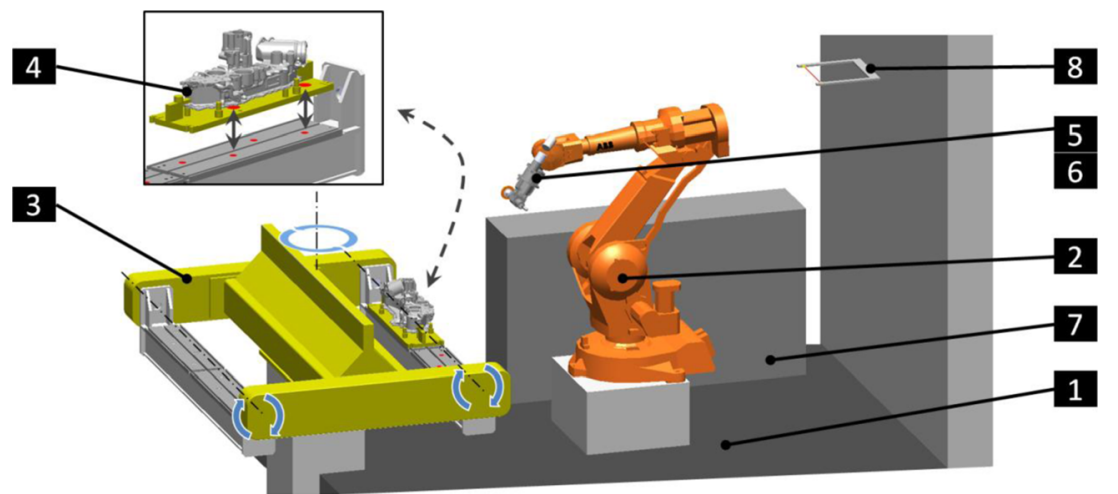


Fig. 8 Touch probe model
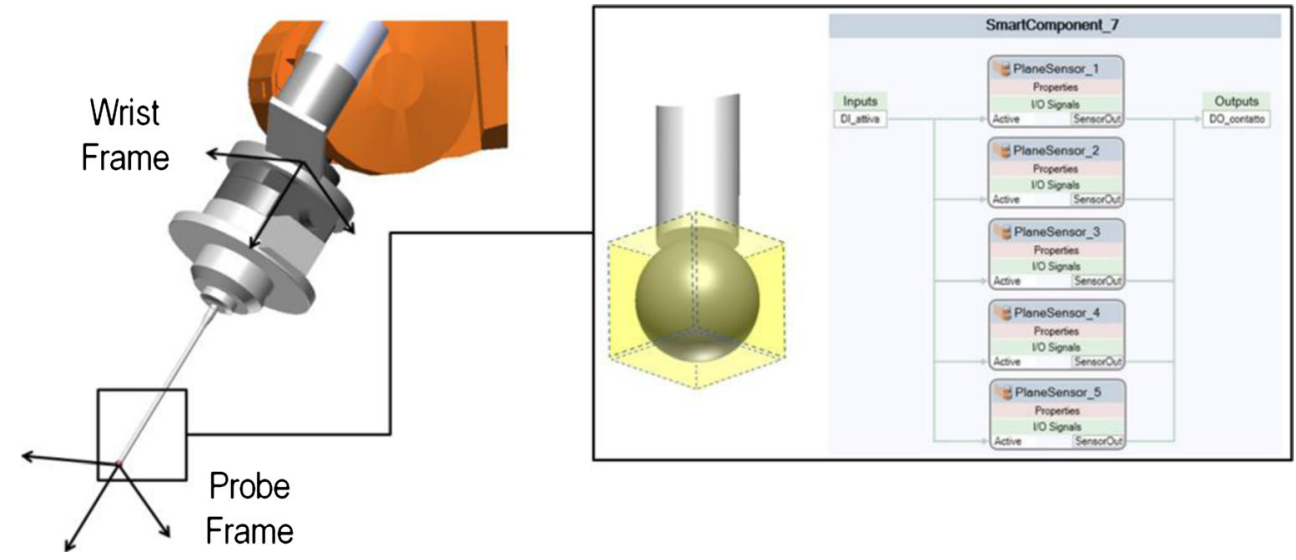

\section{Experimental validation}

The proposed method is employed in the design of a changeable robotic workcell for the accurate finishing of housings of aerospace gear transmissions. The aluminium workpieces range from an envelope of $4 \times 10^{-3} \mathrm{~m}^{3}$ to $8 \times 10^{-2} \mathrm{~m}^{3}$. A part is shown in Fig. 6 before and after machining.

Figure 7 shows the workcell layout. An industrial robot ABB IRB 2400/16 machines a workpiece positioned and oriented by controlled tilting frames on an indexed feeding system. Each workpiece is posed and blocked on the fixtures thanks to a customised set of reference pins. A change system allows a quick replacement of the robot end effectors from a rack. A Renishaw RP1 inspection probe with a ruby ball stylus is the onboard sensor. The stationary sensor is a fork light barrier.

The calibration method is implemented in ABB RobotStudio 5.14.03. The measuring sensors are modeled through smart functions to simulate, optimise and program the calibration tasks, as in [7].

For instance, five IOs gates, shown in Fig. 8, replicate the touch action in the simulation and communicate to the robot controller. Then, the virtual controller stops the robot motors in an event of probe deflection. The subprogram called from the workcell program main is included in the model and can be easily reused in future simulations. The simplification of the virtual contact surface from a sphere to a cube depends on reduced availability of modeling features, but it is suitable for cycle simulation and program generation.

The workpiece frame alignment relies on measurements of a set of target frames on a planar surface and two holes. Thus, the virtual controller computes all the calibrations and updates the wobjdata, corresponding to the data type for frame locations in the ABB RAPID native language. Figure 9 shows an offline simulation of the workpiece alignment with the onboard sensor (a) compared to the online one (b) and the calibration of a finishing disc though the stationary sensor (c).

The OLP of the robot finishing paths and the following online parameters' adjustment provide the accuracy required for the workpiece finishing, namely for breakedge of machined to machined intersections by a minimum chamfer of $0.7 \mathrm{~mm}$. Since the touch probe is involved in the inspection, the workpiece alignment depends on the robot repeatability and accuracy, about $\pm 0.03 \mathrm{~mm}$ for the ABB IRB 2400/16 [29]. Also, the automated alignment avoids the unpredictable errors of the manual one, in most cases, measured $\pm 0.1 \mathrm{~mm}$ accurate and close to $\pm 0.05 \mathrm{~mm}$ only in case of very skilled operators. The accuracy of the automated alignment between finishing paths on the workpiece edges returns a controlled quality, also when the workpiece geometry differs by some millimetres with respect to the nominal one. In Fig. 10, two measuring results on the aerospace workpiece borders machined with the
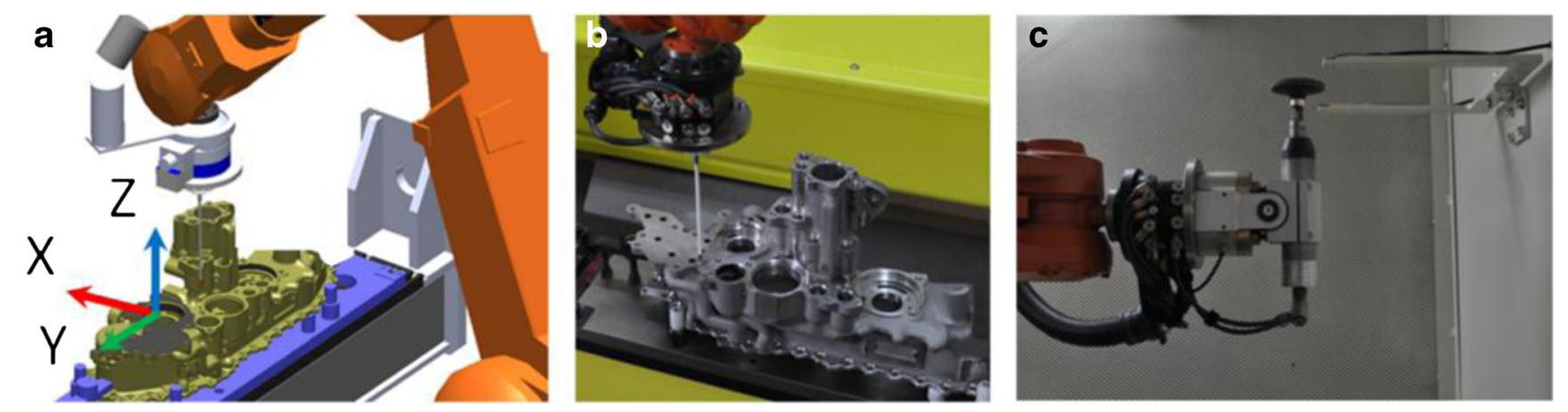

Fig. 9 OLP (a) of online workpiece (b) and tool calibration (c) 
Fig. 10 A gear transmission housing finished
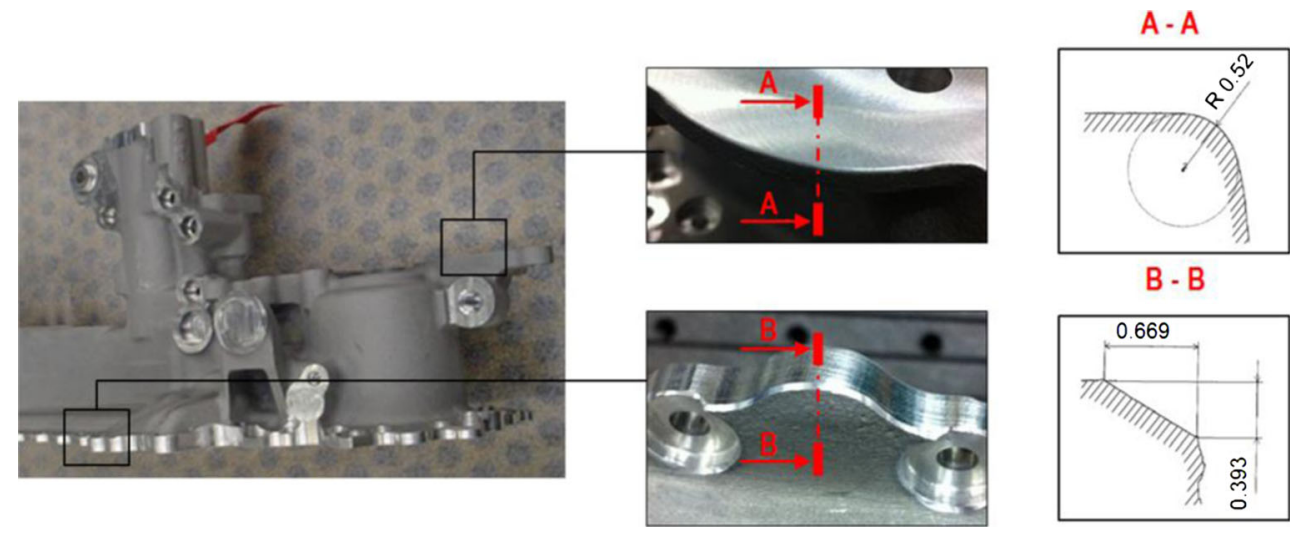

robotic approach confirm that the border sections satisfy the design tolerances, as previously specified.

Finally, Table 2 reports the time suggested between the two following calibration tasks to address a control on the error drifts.

\section{Conclusions}

The aerospace industry requires small lot production of complex parts with high quality specifications. In particular, high accuracy in machining and finishing processes is a demand. Robotic workcells could satisfy the requirements in machining complexity. A design method based on the development of a virtual prototype enables the workcell changeability, focusing on modularity of mechatronic devices and robots programs. Nevertheless, robotic workcells deliver insufficient accuracies for finishing operations, so it is essential to correct the different error sources.

The article proposes a new workcell calibration method consisting of four steps. The calibration of the robot measuring system and the calibration of the peripheral

Table 2 Time set between calibration tasks for the elements in the robotic workcell layout

\begin{tabular}{|c|c|c|c|}
\hline Index & Element & $\begin{array}{l}\text { Workpiece-dependent } \\
\text { (D) independent (I) }\end{array}$ & $\begin{array}{l}\text { Time between } \\
\text { calibration tasks }\end{array}$ \\
\hline 1 & Workcell iron floor & I & - \\
\hline 2 & Industrial robot & I & - \\
\hline 3 & $\begin{array}{l}\text { Workpiece feeding } \\
\text { system }\end{array}$ & I & $1 /$ week \\
\hline 4 & $\begin{array}{l}\text { Fixtured } \\
\quad \text { workpiece }\end{array}$ & $\mathrm{D}$ & Each cycle \\
\hline 5 & Machining tool & $\mathrm{D}$ & $2 / \mathrm{h}$ \\
\hline 6 & $\begin{array}{l}\text { Renishaw RP1 } \\
\text { inspection } \\
\text { probe }\end{array}$ & I & $1 /$ day \\
\hline 7 & Rack & I & - \\
\hline 8 & Stationary sensor & I & 1/day \\
\hline
\end{tabular}

elements reduce the errors due to the workcell devices. The workpiece alignment and the calibration of the robot tools reduce the errors due to the workpiece variation and the process. Each task is run with specific time frames to prevent the errors drifts up to each single processed workpiece alignment.

The major novelty is that the method fully integrates the calibration tasks within a engineering design framework, i.e. finishing strategy validation, process simulation, OLP and robot path generation and robot code commissioning. The virtual prototype integrates the $\mathrm{CAD} 3 \mathrm{D}$ geometries of the devices controlled by the software logics. These first attempt calibration tasks are finally online tuned in the actual workcell to address the actual errors.

The case study on a robotic cell for high quality finishing of aerospace parts proved the feasibility of the calibrations as fully automatic tasks in the robotic process. The accuracy in finishing operations matches the specifications without lowering the productivity of the entire system. An online tuning in the real workcell finely assesses the finishing errors and, at the same time, avoids useless overcorrections. Experimental results prove the method effectiveness in enhancing accuracy in robot machining. According to the test realised, the automated workpiece alignment produces a reduction in workpiece frame location errors from \pm 1 to $\pm 0.1 \mathrm{~mm}$. Moreover, the mismatch between the expected location and the actual location of each workpiece is self-recognised and managed by the system. Such behaviour ensures a constant machining accuracy for every lot even after prolonged stops. The scheduling of calibration time frames enables a constant control on robot, tools and equipment and maintains the path accuracy aligned with the performance offered by a standard industrial robot.

Acknowledgments The authors want to express their gratitude to L. Passoni, D. Passoni and L. Ferrari from SIR S.p.A. (Modena, Italy) for their technical and managerial contribution to the project and AVIO S.p.A. (Torino, Italy) for supporting the experimental tests. 


\section{References}

1. Jayaweera N, Webb P (2010) Robotic edge profiling of complex components. Ind Robot Int J 38(1):38-47. doi:10.1108/ 01439911111097832

2. Lehmann C, Pellicciari M, Drust M, Gunnink JW (2013) Machining with industrial robots: the COMET project approach. In: International Conference on Flexible Automation and Intelligent Manufacturing (FAIM), pp. 27-36, Porto, Portugal

3. Chen Y, Dong F (2013) Robot machining: recent development and future research issues. Int J Adv Manuf Technol 66(9-12):1489 1497. doi:10.1007/s00170-012-4433-4

4. Milutinovic D, Glavonjic M, Slavkovic N, Dimic Z, Zivanovic S, Kokotovic B, Tanovic L (2011) Reconfigurable robotic machining system controlled and programmed in a machine tool manner. Int $\mathbf{J}$ Adv Manuf Technol 53(9-12):1217-1229. doi:10.1007/s00170-0102888-8

5. ElMaraghy HA (2005) Flexible and reconfigurable manufacturing systems paradigms. Int J Flex Manuf Syst 17(4):261-276. doi:10. 1007/s10696-006-9028-7

6. Bi ZM, Lang SYT (2005) General modular robot architecture and configuration design. In: Proc IEEE Int Conf Mechatron Automat, 1 pp 268-273, Niagara Falls, Ontario, Canada. doi:10.1109/ICMA. 2005.1626558

7. Pellicciari M, Leali F, Andrisano AO, Pini F (2012) Enhancing changeability of automotive hybrid reconfigurable systems in digital environments. Int J Interact Des Manuf 6(4):251-263. doi:10.1007/ s12008-012-0156-x

8. Andrisano AO, Leali F, Pellicciari M, Pini F, Vergnano A (2012) Hybrid reconfigurable system design and optimization through virtual prototyping and digital manufacturing tools. Int J Interact Des Manuf 6(1):17-27. doi:10.1007/s12008-011-0133-9

9. Pan Z, Polden J, Larkin N, Van Duin S, Norrish J (2012) Recent progress on programming methods for industrial robots. Robot Comput Integr Manuf 28(2):87-94. doi:10.1016/j. rcim.2011.08.004

10. Li X, Zhang B (2011) Toward general industrial robot cell calibration. In: Proc 2011 5th IEEE Int Conf Robot Autom Mechatron (RAM), pp 137-142. Qingdao, China. doi:10.1109/ RAMECH.2011.6070470

11. Lu T, Lin GCI (1997) An on-line relative position and orientation error calibration methodology for workcell robot operations. Robot Comput Integr Manuf 13(2):89-99. doi:10.1016/ S0736-5845(96)00029-4

12. Pan Z, Zhang H (2009) Improving robotic machining accuracy by real-time compensation. In: Proc ICROS-SICE Int Joint Conf 2009 (ICCAS-SICE 2009), pp 4289-4294, Fukuoka, Japan

13. Kurt M, Bagci E (2011) Feedrate optimisation/scheduling on sculptured surface machining: a comprehensive review, applications and future directions. Int J Adv Manuf Technol 55(9-12):1037-1067. doi:10.1007/s00170-010-3131-3
14. Pan Z, Zhang H, Zhu Z, Wang J (2006) Chatter analysis of robotic machining process. J Mater Process Technol 173(3):301-309. doi:10. 1016/j.jmatprotec.2005.11.033

15. Cherif M, Knevez JY, Ballu A (2010) Thermal aspects on robot machining accuracy. In: Proc IDMME-Virtual Concept 2010, 3, Bordeaux, France

16. Kawahara S, Ohishi K, Miyazaki T, Yokokura Y (2013) Vibration suppression feedback control on angular transmission error of cycloid gear for industrial robot. In: Proc 2013 I.E. Int. Conf. Mechatron (ICM 2013), pp 859-864. Vicenza, Italy. doi:10.1109/ICMECH. 2013.6519153

17. Ruderman M, Hoffmann F, Bertram T (2009) Modeling and identification of elastic robot joints with hysteresis and backlash. IEEE Trans Ind Electron 56(10):3840-3847. doi:10.1109/TIE.2009. 2015752

18. Siciliano B, Kathib O (2008) Handbook of robotics. Springer, New York. doi:10.1007/978-3-540-30301-5

19. Shiakolas PS, Conrad KL, Yih TC (2002) On the accuracy repeatability and degree of influence of kinematics parameters for industrial robots. Int J Model Simul 22(3):1-10

20. Mustafa SK, Pey YT, Guilin Y, I-Ming C (2010) A geometrical approach for online error compensation of industrial manipulators. In: Porc 2010 IEEE/ASME Int Conf Adv Intell Mechatron, pp 738-743, Montreal, Ontario, Canada. doi:10.1109/AIM.2010.5695784

21. Lim HK, Kim DH, Kim SR, Kang HJ (2009) A practical approach to enhance positioning accuracy for industrial robots. In: Proc ICROSSICE Int Joint Conf 2009 (ICCAS-SICE 2009), pp 2268-2273, Fukuoka, Japan

22. Mooring BW, Roth ZS, Driels MR (1991) Fundamentals of manipulator calibration. Wiley, New York

23. Elatta AY, Gen LP, Zhi FL, Daoyuan Y, Fei L (2004) An overview of robot calibration. Inf Technol J 3(1):74-78. doi:10.3923/itj.2004.74.78

24. Gan Z, Sun Y, Tang Q (2004) In-process relative robot workcell calibration. US Patent 6,812,665

25. Cheng FS (2007) The method of recovering robot TCP positions in industrial robot application programs. In: Proc 2007 I.E. Int Conf Mechatron and Autom (ICMA 2007), pp 805-810. doi:10.1109/ ICMA.2007.4303648

26. Bley H, Bernerdi M, Franke C, Seel U (2001) Process-based assembly planning using a simulation system with cell calibration. In: Proc 2001 I.E. Int Symp Assem Task Plan (ISATP 2001), pp 116-121, Fukuoka, Japan. doi:10.1109/ISATP.2001.928976

27. Ribeiro F, McMaster R (1997) A low cost cell calibration technique and its PC based control software. In: Proc 1997 I.E. Int Symp Ind Electron (ISIE 1997), 3 pp 840-845, Guimaraes, Portugal. doi:10. 1109/ISIE.1997.648649

28. Pellicciari M, Andrisano AO, Leali F, Vergnano A (2009) Engineering method for adaptive manufacturing systems design. Int J Interact Des Manuf 3(2):81-91. doi:10.1007/s12008-009-0065-9

29. ABB Product Specification Articulated Robot IRB 24003HAC9112-1, Rev. T 